Supporting Information:

\title{
Rational Design of Stimuli-Responsive Polymers Modified Nanopores for Selective and Sensitive Determination of Salivary Glucose
}

Miao Yang, Chunrong Ma, Shushu Ding, Yujie Zhu, Guoyue Shi,* Anwei Zhu*

School of Chemistry and Molecular Engineering, Shanghai Key Laboratory for Urban

Ecological Processes and Eco-Restoration, East China Normal University, 500

Dongchuan Road, Shanghai 200241, People's Republic of China

*To whom correspondence should be addressed.

E-mail: awzhu@chem.ecnu.edu.cn

Tel: +86-21-54340042; Fax: +86-21-54340042 


\section{Table of contents}

1. SEM images of the unmodified glass nanopore tip (Figure S1)

2. I- $V$ curve of the unmodified glass nanopore (Figure $\mathrm{S} 2$ )

3. TEM image of bare glass nanopore tip (Figure S3)

4. FTIR spectra of the monomers and copolymer (Figure S4)

5. ${ }^{1} \mathrm{H}$ NMR spectrum of PATPBA-co-PNIPAAm (Figure S5)

6. XPS spectra (Figure S6)

7. Repeatibility (Figure S7)

8. Control experiments (Figure S8)

9. The molecular structures of the five monosaccharides (Figure S9)

10.Comparison of PAPBA-co-PNIPAAm and PATPBA-coPNIPAAm in saccharides selectivity (Figure S10)

11.Comparison of the performance of different PBA-based glucose detection methods (Table S1)

12. Molecular sturecture of the polymers in this study (Scheme S1) 
1. SEM images of the unmodified glass nanopore tip
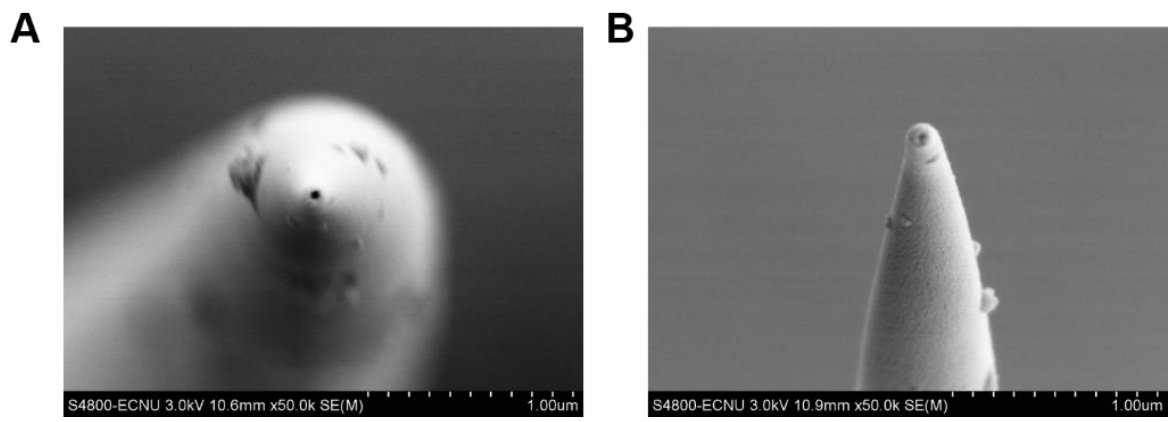

Figure S1. SEM images of the unmodified glass nanopore tip from different angles. 


\section{I-V curve of the unmodified glass nanopore}

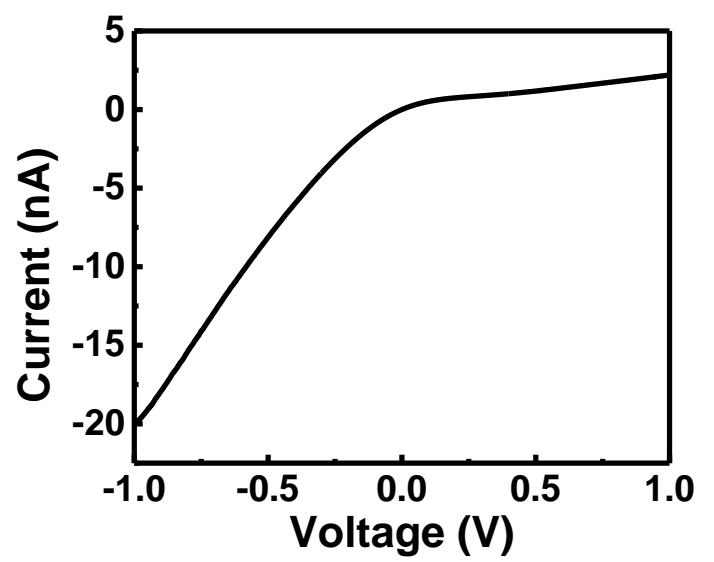

Figure S2. I-V curve of the unmodified glass nanopore corresponding to the image in Figure S1. Both the external bulk solution and the solution backfilled in the nanopore was $0.1 \mathrm{M} \mathrm{KCl}$.

The orifice diameter of the glass nanopore could be estimated from electrochemical measurements based on eqn (1).

$$
r=\frac{1}{\pi \kappa R \tan \frac{\theta}{2}}
$$

Here, $\mathrm{r}$ is the orifice radius of glass nanopore; $\kappa$ is the conductivity of the electrolyte solution $(1.2 \mathrm{~S} / \mathrm{m}$ for $0.1 \mathrm{M} \mathrm{KCl}) ; \mathrm{R}$ is the measured resistance of the nanopore and $\theta$ is the cone angle. The nanopore radius was estimated to be $\sim 35 \mathrm{~nm}$ from the electrochemical measurement, which was consistent with the SEM image. 
3. TEM image of bare glass nanopore tip

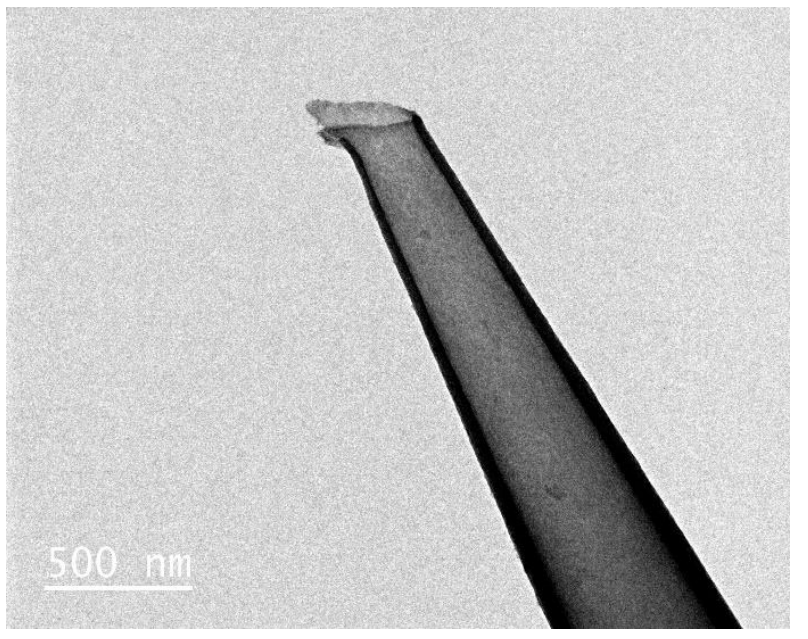

Figure S3. TEM image of the unmodified (Au-free) glass nanopore tip. 
4. FTIR spectra of the monomers and copolymer

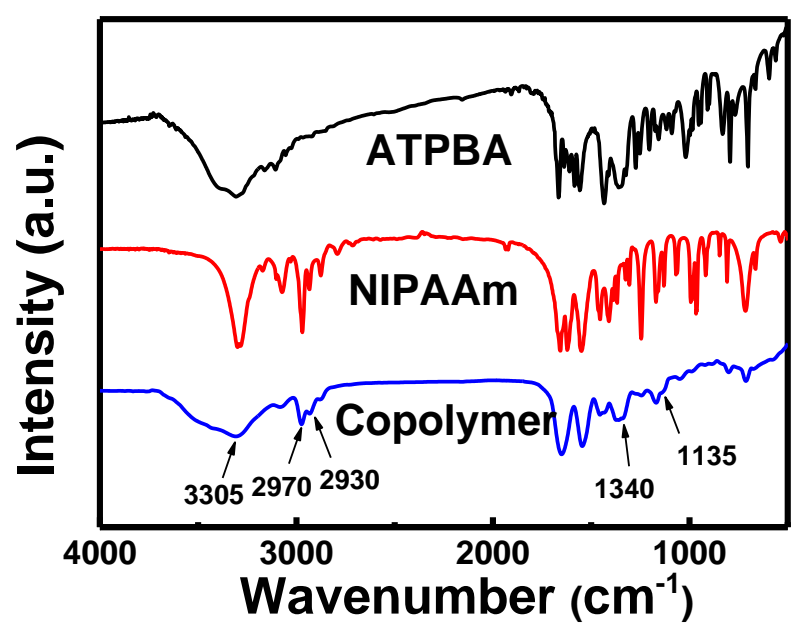

Figure S4. FTIR spectra of ATPBA (black line), NIPAAm (red line) and PATPBA-co-PNIPAAm (blue line). 


\section{5. ${ }^{1}$ H NMR spectrum of PATPBA-co-PNIPAAm}
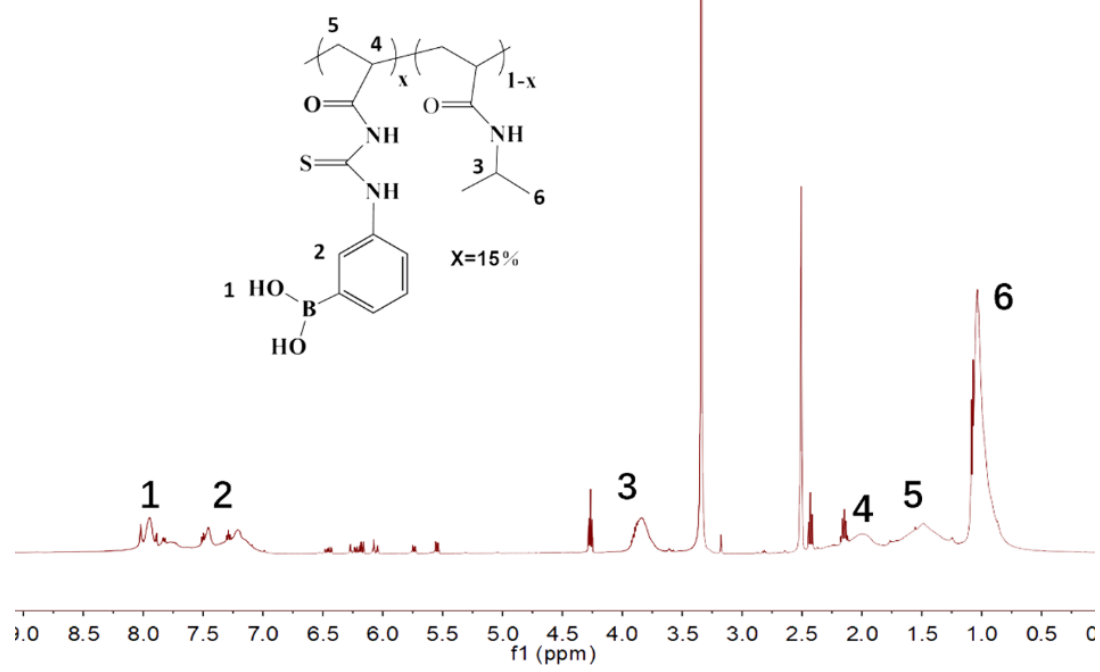

Figure S5. ${ }^{1} \mathrm{H}$ NMR spectrum of PATPBA-co-PNIPAAm in d6-DMSO. The signal at $8.01 \mathrm{ppm}$ can be assigned to two protons of $\mathrm{B}(\mathrm{OH})_{2}$ in $\mathrm{PBA}$ unit. The broad peaks at 7.0-8.0 ppm correspond to the protons of $\mathrm{CH}$ in phenyl group of PBA. The peaks at $3.82 \mathrm{ppm}$ and $1.02 \mathrm{ppm}$ are attributed to one proton of $\mathrm{CH}\left(\mathrm{CH}_{3}\right)_{2}$ and six protons of $\mathrm{CH}\left(\mathrm{CH}_{3}\right)_{2}$ in $\mathrm{PNI}$ unit. The chemical shifts of methine protons $(\mathrm{CH})$ and methylene protons $\left(\mathrm{CH}_{2}\right)$ in the backbone appear at $1.98 \mathrm{ppm}$ and $1.48 \mathrm{ppm}$. All the results indicate that the copolymer has been successfully synthetized. 
6. XPS spectra
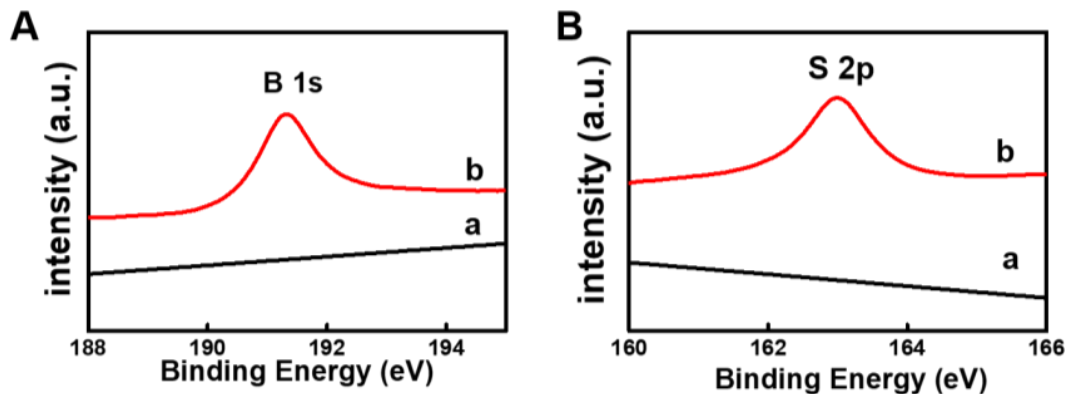

Figure S6. XPS spectra of (A) B 1s and (B) S 2p peaks for (a) the Au-coated planar glass slide and (b) the copolymers-modified glass slide. 


\section{Repeatibility}

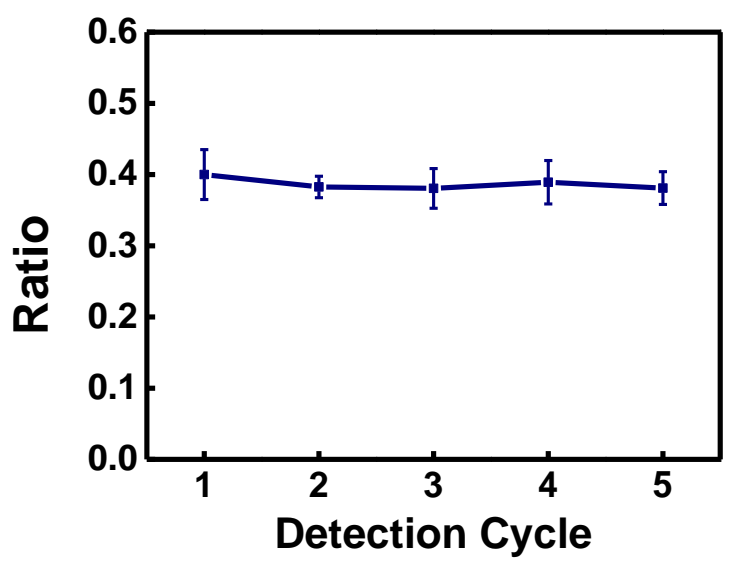

Figure S7. The ionic current change ratios at $-1.0 \mathrm{~V}$ of the copolymer functionalized nanopore used for several repeated detection of $10^{-7} \mathrm{M}$ glucose in $10 \mathrm{mM} \mathrm{KCl}$ solution buffered with $5 \mathrm{mM}$ carbonate buffer solution ( $\mathrm{pH} 9.0$ ). After each glucose detection, the nanopore surface was renewed by the treatment of $10 \mathrm{mM} \mathrm{KCl}$ solution $(\mathrm{pH} \mathrm{7.0)}$ for $10 \mathrm{~min}$. 


\section{Control experiments}
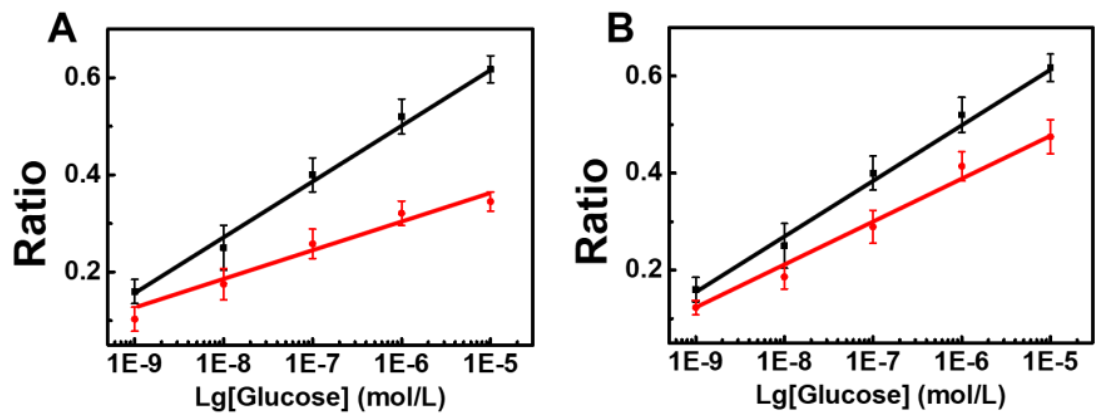

Figure S8. Curves of the ionic current change ratios at $-1.0 \mathrm{~V}$ for nanopores changing with concentrations of glucose. (A) The effect of the functional switching units poly( $N$-isopropylacrylamide) backbon. Black square: PATPBAco-PNIPAAm modified glass nanopores; Red circle: PATPBA-modified glass nanopores. (B) The effect of $\mathrm{pH}$ on the current change ratios of PATPBA-coPNIPAAm-modified glass nanopores. Black square: $\mathrm{pH}$ 9; red circle: $\mathrm{pH} 7$. 
9. The molecular structures of the five monosaccharides
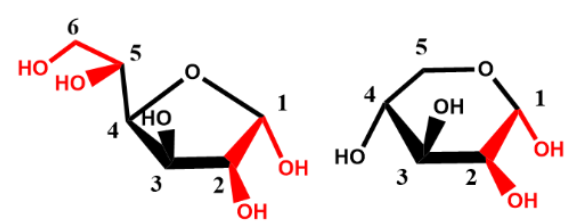

D-Glucose

D-Xylose

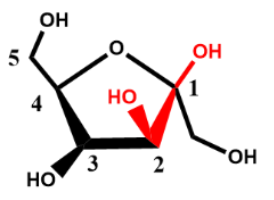

D-Fructose

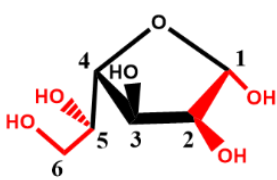

D-Galactose

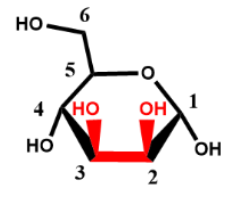

D-Mannose

Figure S9. The molecular structures of the five monosaccharides: D-glucose, D-xylose, D-galactose, D-fructose and D-mannose. 


\section{Comparison of PAPBA-co-PNIPAAm and PATPBA-co-PNIPAAm}

in saccharides selectivity

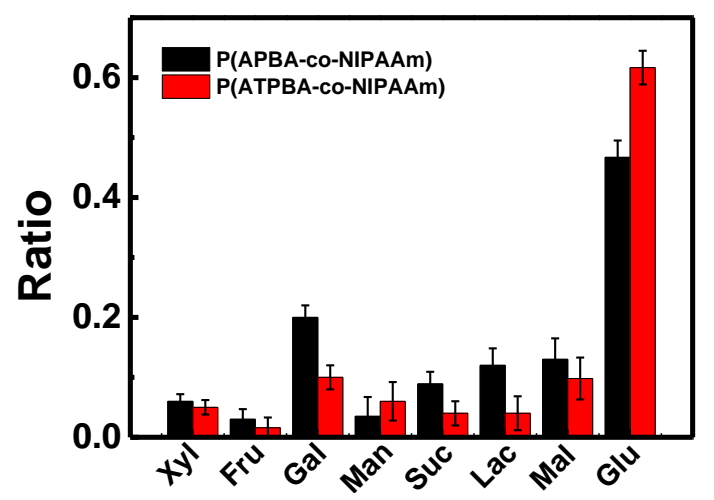

Figure S10. Effect of thiourea groups on the selectivity of copolymer modified nanopores. Saccharides: xylose, fructose, galactose, mannose, sucrose, lactose, maltose, glucose. $10 \mu \mathrm{M}$ for glucose, $10 \mu \mathrm{M}$ for other saccharides; Left (black): PAPBA-co-PNIPAAm modified glass nanopores; Right (red): PATPBAco-PNIPAAm modified glass nanopores. 


\section{Table S1 Comparison of the performance of different PBA-based}

\section{glucose detection methods}

\begin{tabular}{|c|c|c|c|c|c|c|}
\hline Sensing materials & Technique & Detection limit $(\mu \mathrm{M})$ & Linear range $(\mu \mathrm{M})$ & Real sample & Measurement time $\mathrm{e}^{\mathrm{a}}$ & References \\
\hline $\mathrm{APBA} / \mathrm{rGO}$ & EIS & 30 & $1 \times 10^{2}-5 \times 10^{4}$ & Serum & $35 \mathrm{~min}$ & 1 \\
\hline $\begin{array}{l}\text { Pyrene-1-boronic } \\
\text { acid/ carbon } \\
\text { nanotube transistor }\end{array}$ & Electricity & 0.3 & $1-1 \times 10^{5}$ & No & - & 2 \\
\hline $\begin{array}{c}\mathrm{PBA} / \text { glassy carbon } \\
\text { (GC) }\end{array}$ & EIS & - & $0-5 \times 10^{4}$ & No & - & 3 \\
\hline $\mathrm{MIP} / \mathrm{pTBA} / \mathrm{AuNPs}$ & Potentiometric & $1.9( \pm 0.15) \times 10^{-1}$ & $3.2 \times 10^{-1}-1.0 \times 10^{3}$ & Saliva & not mentioned & 4 \\
\hline $\begin{array}{l}\text { PBA-modified } \\
\text { CdTe/ZnTe/ZnS } \\
\text { CSS QDs }\end{array}$ & Fluorescence & 300 & $4 \times 10^{2}-2 \times 10^{4}$ & Living cells & $6 \mathrm{~h}$ & 5 \\
\hline $\begin{array}{l}\text { PATPBA-co- } \\
\text { PNIPAAm }\end{array}$ & Ionic Current & $1 \times 10^{-3}$ & $1 \times 10^{-3}-10$ & Saliva & $\sim 26 \min$ & This work \\
\hline
\end{tabular}

a Measurement time is the total time for measuring a real sample, including the time of preprocessing of sample and the response time. 
12. Scheme S1 Molecular sturecture of the polymers in this study

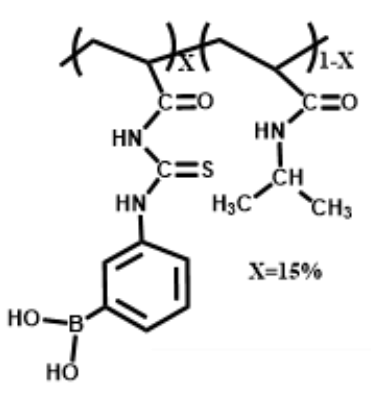

PATPBA-CO-PNIPAAm

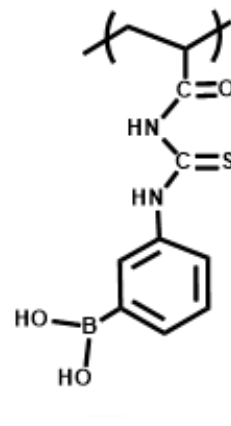

PATPBA

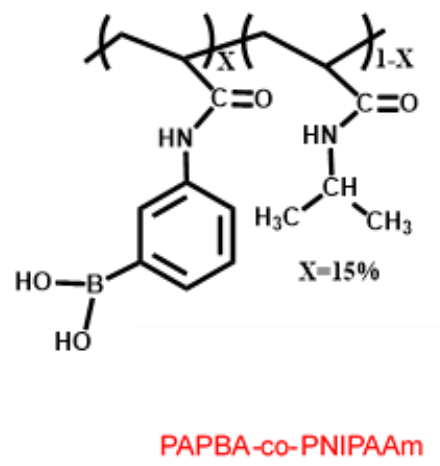




\section{REFERENCES}

1. Li, S.; Zhang, Q.; Lu, Y.; Ji, D.; Zhang, D.; Wu, J.; Chen, X.; Liu, Q. Sens. Actuat. B: Chem. 2017, 244, 290-298.

2. Lerner, M. B.; Kybert, N.; Mendoza, R.; Villechenon, R.; Bonilla Lopez, M. A.; Charlie Johnson, A. T. Appl. Phys. Lett. 2013, 102, 183113.

3. Morita, K.; Hirayama, N.; Imura, H.; Yamaguchi, A.; Teramae, N. J. Electroanal. Chem. 2011, 656, 192-197.

4. Kim, D. M.; Moon, J. M.; Lee, W. C.; Yoon, J. H.; Choi, C. S.; Shim, Y. B. Biosens. Bioelectron. 2017, 91, 276-283.

5. Wu, W.; Zhou, T.; Berliner, A.; Banerjee, P.; Zhou, S. Angew. Chem., Int. Ed. 2010, $49,6554-6558$. 